\title{
Radio over Fiber Link Design for Next Generation Wireless Systems
}

\author{
David Wake, Anthony Nkansah and Nathan J. Gomes, Senior Member, IEEE
}

(Invited Paper)

\begin{abstract}
The performance of radio over fiber (RoF) links using low cost optoelectronic components is assessed for distributed antenna applications in next generation wireless systems. Important design issues are discussed and an example link design is presented for a wireless system requiring the transmission of four radio channels per link direction, each with $100 \mathrm{MHz}$ bandwidth, modulation complexity of 256-QAM and 2048 OFDM subcarriers. We show that the noise introduced by the RoF links does not have a significant impact on wireless range, provided the wireless system has uplink power control. Finally, we compare the cost and performance of RoF links for this application with alternative link types that use digitized radio transmission and show that $\mathrm{RoF}$ is the optimum choice from a cost perspective.
\end{abstract}

Index Terms-Distributed antenna systems, next generation wireless systems, radio over fiber

\section{INTRODUCTION}

$\mathrm{R}$ adio over fiber (RoF) is a well established technique for the distribution of wireless communications signals [1, 2]. One of the main applications is known as the distributed antenna system (DAS). In a DAS, RoF transmission links are used to connect a central unit (CU) containing the radio base stations to a number of remote antenna units (RAUs), in order to provide excellent coverage and dedicated capacity for short range communications in areas such as city centers, office blocks, shopping malls and transport hubs [3]. Fig. 1 shows the DAS concept for the case of a city center application. Here, the CU and the base stations are co-located in a building, sometimes known as a base station hotel, and RoF links are used to connect the $\mathrm{CU}$ to the RAUs within the coverage area. The RAUs are located in items of street furniture such as lamp posts and also on the sides of buildings.

RoF uses analog rather than digital optical transmission and therefore suffers from impairments due to noise and distortion. The performance of these analog optical

Manuscript received November 15, 2009. This work was partially supported by the European Commission under Grant Agreement No. FP7ICT-2007-215533 (FUTON).

D. Wake, A. Nkansah and N. J. Gomes are with the Broadband and Wireless Communications Group, University of Kent, Canterbury, CT2 7NT, UK. (phone: +44 1227 823719, email: n.j.gomes@kent.ac.uk). transmission links, even when using low cost semiconductor lasers and photodiodes, is generally acceptable for present generations of wireless communications systems such as $2^{\text {nd }}$ generation (2G), for example GSM (Global System for Mobile) and $3^{\text {rd }}$ generation (3G), for example UMTS (Universal Mobile Telecommunications System).

Next generation wireless systems will have more demanding noise and distortion requirements for the RoF links used in DAS applications. This is because, to achieve higher data throughput, these future wireless systems will require a number of measures to be used [4]. For example, wider channel bandwidth and higher level modulation schemes are likely to be employed. Orthogonal frequency division multiplexing (OFDM) will be used to provide a robust wireless channel and, probably, the basis for a multiple access scheme (OFDMA). Throughput may be increased further by the use of multiple input/multiple output (MIMO) antennas, which increases the number of radio channels that must be transported by the RoF links.

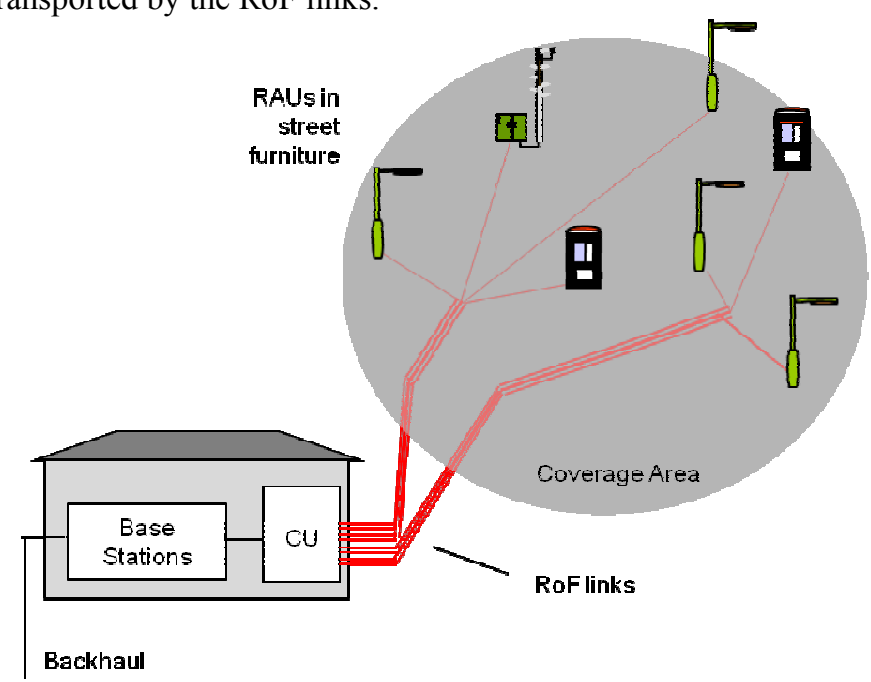

Fig. 1. Distributed antenna system using radio over fiber for a city center application.

For example, the 3rd Generation Partnership Project (3GPP) has recently specified LTE (Long Term Evolution) as a step towards $4 \mathrm{G}$ systems [5]. LTE will have peak downlink user rates of around $100 \mathrm{Mb} / \mathrm{s}$ using a channel bandwidth of up to $20 \mathrm{MHz}$, modulation complexity up to 64-QAM, 2048 subcarrier OFDM and MIMO with up to $4 \times 4$ antennas. It is 
envisaged that true $4 \mathrm{G}$ systems will have peak downlink data rates of around $1 \mathrm{~Gb} / \mathrm{s}$ and a number of candidate radio interfaces are under consideration to achieve this target. The $3 \mathrm{GPP}$ effort in this area is LTE-Advanced, and it is expected that channel bandwidths of up to $100 \mathrm{MHz}$ will be required.

FUTON (an EU Framework 7 project) provides an example of a $4 \mathrm{G}$ radio interface [6]. This project is investigating the capabilities of a DAS architecture with a high throughput radio interface, known as the distributed broadband wireless system (DBWS). It envisages a physical layer with a throughput capability of up to $1 \mathrm{~Gb} / \mathrm{s}$. To achieve this throughput, channel bandwidths up to $100 \mathrm{MHz}$ and modulation levels up to 256-QAM are likely to be required. OFDMA with up to 2048 subcarriers and various MIMO configurations are also under investigation in this project.

All of these requirements have major implications for the performance and design of the RoF links that connect the CU to the RAUs in a DAS architecture. This paper investigates the performance of the RoF links in the light of these requirements and looks at whether low cost lasers and photodiodes can still be used. The paper also considers whether RoF is still the preferred option for these systems in the light of recent open base station specifications such as the Common Public Radio Interface (CPRI) [7] and the Open Base Station Architecture Initiative (OBSAI) [8]. These systems have base stations with a digital output (digitized radio) and use digital transmission between the $\mathrm{CU}$ and RAUs. Note that transmission of the digital baseband $1 \mathrm{~Gb} / \mathrm{s}$ data signals is not considered in this paper. Such a scheme is not appropriate for this application because of the added complexity required in the remote units and more importantly because MIMO processing functions such as maximum ratio combining cannot be achieved with demodulated signals.

\section{RADIO OVER FIBER LINK DESIGN ISSUES}

This section looks at each of the major issues affecting the performance and design of RoF links that are raised by the requirements of next generation wireless communications systems.

\section{A. Carrier Frequency}

This is a very important parameter for RoF links that do not utilize frequency translation, i.e., where transmission over the link is at the radio carrier frequency, because it determines the type of optoelectronic components that can be used. Low cost semiconductor lasers are readily available with an upper modulation frequency limit of around $3 \mathrm{GHz}$. Beyond that, semiconductor lasers become more and more expensive, up to around $20 \mathrm{GHz}$, after which they are no longer commercially available. Thus, above $20 \mathrm{GHz}$, the use of external optical modulators becomes essential, but this is an expensive option.

Next generation wireless systems are likely to use bands below $6 \mathrm{GHz}$, where radio propagation characteristics are suitable for low cost network deployments, although higher frequencies may be preferred in some cases due to greater spectrum availability. If higher frequencies are used, for example the mm-wave bands, fiber dispersion can become a serious problem even over relatively short spans for conventional double sideband modulation. Advanced modulation and transmission schemes must be employed which will lead to higher cost. Very high bit rate RoF links have been demonstrated at mm-wave frequencies [9], but these have been mostly for very short range personal area network applications where very high dynamic range is not required. An attractive solution to this problem is to use frequency translation, where the signals are carried over the RoF link at a much lower frequency, or intermediate frequency (IF). IF transmission has several important advantages:

i) it allows the use of low frequency, low cost components,

ii) it allows the use of a simple intensity modulation direct detection transmission scheme and direct modulation of semiconductor laser diodes,

iii) it allows multiple channels to be transmitted over a single link at the same radio carrier frequency, for example, to carry multiple MIMO channels.

These advantages make IF transmission a compelling choice for situations where the radio carrier frequency is higher than $3 \mathrm{GHz}$ or where several radio channels must be transported simultaneously over a single link. Frequency translation is relatively straightforward to implement at low cost and a low frequency reference tone can be transported over the RoF link to ensure precise frequency locking at the remote site.

\section{B. Radio Channel Bandwidth}

As mentioned previously, channel bandwidths of up to 100 $\mathrm{MHz}$ are being envisaged for next generation wireless systems to meet the user data rate requirements. Channel noise scales directly with bandwidth, which means that higher signal power is needed to maintain the minimum carrier to noise ratio $(C N R)$ required for successful demodulation. The increased channel noise leads to reduced receiver sensitivity and therefore reduced radio range.

This is the true for the intrinsic wireless system, i.e. without RoF links connecting the radio base stations and antennas. The RoF links must be capable of supporting the required CNR in the face of higher channel noise, so must have good performance. The SFDR of the RoF link scales inversely with channel bandwidth with a factor of $2 / 3$, so increasing the bandwidth from $10 \mathrm{MHz}$ to $100 \mathrm{MHz}$ results in a SFDR reduction of $6.7 \mathrm{~dB}$.

\section{Number of Channels}

RoF links have a maximum composite input power limit beyond which the signal quality is degraded to unacceptable levels. All channels must share this composite power, therefore the larger the number of channels, the lower the power per channel becomes. This results in a lower CNR at 
the output of the RoF link, which in general will lead to reduced radio range. In the downlink direction, the reduction in signal power can be compensated by increasing the amplifier gain in the remote unit, but the maximum amplifier gain is limited by two main factors:

i) maximum transmitted noise and spurious emissions requirements of the wireless system specification,

ii) stability requirements in the remote unit, since high gain for both uplink and downlink directions may lead to oscillation.

\section{OFDM}

OFDM signals have high peak-to-average power ratio $(P A P R)$, which results from variations of the relative phase in the subcarriers that combine to give the composite signal. Peak power arises when all of the subcarriers have the same phase. With no amplitude modulation on the subcarriers, such as in the case of QPSK, the maximum PAPR becomes $10 \log (N)$ where $N$ is the number of subcarriers. For example, for 256 subcarriers, the maximum $P A P R$ would be $24 \mathrm{~dB}$. However, this is a very rare occurrence, especially for large $N$, and in practice PAPR is limited to reasonable values by considering an acceptable clipping probability. Using the fact that the complex envelope of the OFDM signal is approximately Rayleigh distributed for large $N$, the probability that the PAPR exceeds a certain threshold $(\gamma)$ is found by solving for the complementary cumulative distribution and for band-limited signals is given by [10]:

$$
P(P A P R \geq \gamma)=1-\exp [-\sqrt{ }(\pi \gamma / 3) N \exp (-\gamma)]
$$

From (1) it is evident that the statistical PAPR does not depend on the QAM level, unlike the maximum PAPR. The expression gives the statistical $P A P R=11.7 \mathrm{~dB}$ and $12.6 \mathrm{~dB}$ for $N=64$ and 2048 respectively for a clipping probability of $10^{-4}[11]$.

To accommodate signals with high $P A P R$, the input power to the RoF link must be reduced by the PAPR, compared to the situation where the input signal has a constant envelope. A convenient method of representing this power reduction is to introduce a back-off factor from the $1 \mathrm{~dB}$ compression point of the link. This back-off factor is likely to be at least as high as the statistical PAPR of the signal for a given probability of clipping. Obviously, some clipping will occur due to the statistical nature of the signal. Clipping will introduce in-band distortion that will reduce the CNR of the signal and out-ofband radiation that may affect neighboring channels or violate the spectral mask requirements. The resulting EVM increase due to in-band clipping noise for the case of Nyquist sampling and a soft limiter nonlinearity (laser biased at low bias) was demonstrated through simulation in [12].

Although the above discussion has considered distortion due to clipping, the large signal (current) amplitudes from an OFDM signal driving a laser will cause distortion due to any device nonlinearity. Back-off will generally reduce the nonlinear distortion. However, a high back-off factor means that the output power and output CNR of the RoF link is reduced. Again, this results in reduced performance in terms of radio range. In the downlink direction, the reduction in performance can be mitigated to a limited extent by increasing the gain of the remote unit amplifier. As mentioned earlier, the maximum amplifier gain is constrained by maximum transmitter noise and remote unit stability requirements.

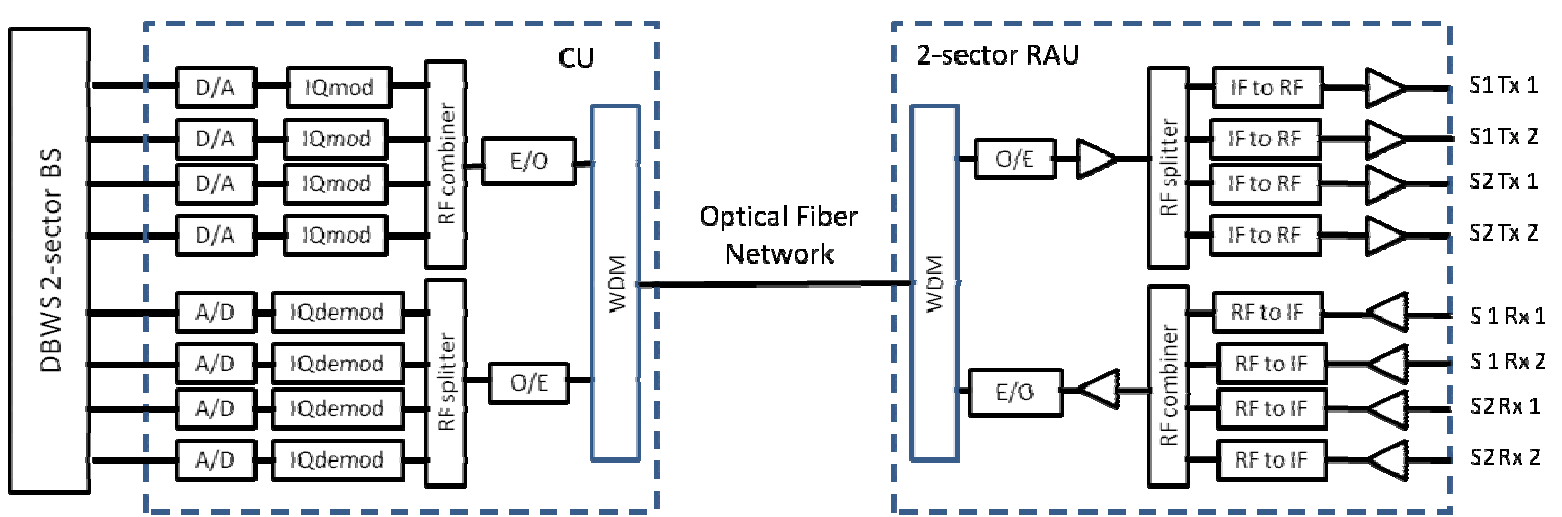

Fig. 2. Simplified DBWS link design. $\mathrm{S} 1$ and $\mathrm{S} 2$ are the two sectors; Tx1, Tx2, Rx1 and Rx 2 are the $2 \times 2$ MIMO channels

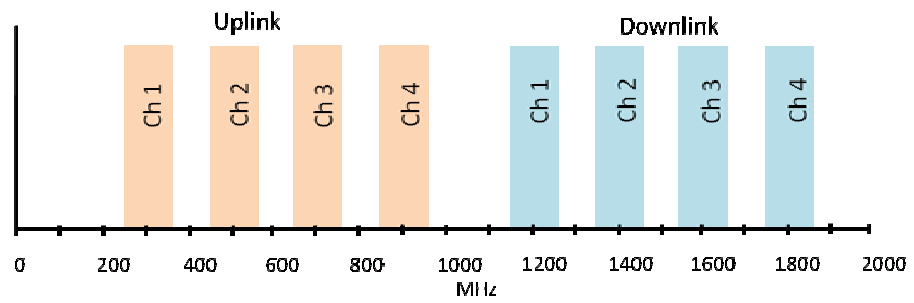

Fig. 3. Frequency plan for SCM transmission system. 


\section{E. Modulation Complexity}

The CNR of the RoF link must be high enough to allow successful demodulation of the signal. As the modulation complexity increases, so the minimum CNR requirement also increases. The minimum CNR also depends on the radio channel characteristics. Predicted CNR requirements are given in [13] for various combinations of modulation scheme, coding rate and channel characteristics. For example, for a Ricean channel and a coding rate of 5/6, the minimum CNR required for quasi-error-free performance (after Reed-Solomon decoding) for 16-QAM and 64-QAM is $14.4 \mathrm{~dB}$ and $20 \mathrm{~dB}$ respectively. A high CNR requirement leads to a reduction in radio range due to desensitization of the radio receiver. This is a feature of the intrinsic wireless system and is not directly related to the performance of the RoF link as long as the link has sufficient CNR at its output. The CNR performance of the RoF link must therefore be increased for high levels of modulation complexity.

\section{F. $M I M O$}

The use of MIMO necessitates the transport of several radio channels between the central unit and the remote units at exactly the same radio carrier frequency. There are several options for providing this functionality:

i) using separate RoF links on separate optical fibers (space division multiplexing, SDM),

ii) using separate RoF links on the same fiber but on separate optical wavelengths (wavelength division multiplexing, WDM), or

iii) using the same RoF link where each channel is frequency translated to a separate IF (subcarrier multiplexing, SCM).

The preferred option will depend upon several factors such as the availability and cost of optical fibers and the configuration of the optical network connecting the central and remote units. In general, optical fiber in metropolitan areas in expensive to install or to lease, so options that minimize the need for fiber will be preferred. Note that the focus of this work is for outdoor applications; for inbuilding applications where fiber installation cost is not so great, the use of separate fibers may be more appropriate. WDM is often used to minimize the need for fiber, but there is a limited number of wavelengths available if the relatively low cost variant, coarse WDM (CWDM) is used. Of the three options given above, SCM is the one that has the ability to minimize the number of fibers in the optical network, particularly in situations where wavelengths must be conserved to enable support of multiple remote units. SCM is relatively simple to implement and is also likely to be lower cost than WDM.

The use of frequency translation in SCM systems means that the radio signals are carried at IF. These frequencies can be chosen to be low enough to allow the use of low cost optoelectronic components. Furthermore, these components generally have better performance at lower frequencies. This aspect gives SCM a further advantage when considering which option to use when transporting multiple MIMO channels.

\section{EXAMPLE RADIO OVER FIBER LINK DESIGN}

\section{A. Link Architecture}

In this section, the design of a RoF link is described that takes the issues discussed in the previous section into account. The FUTON DBWS is used as an example.

The main initial consideration is the type of link architecture required to support multiple MIMO channels. SCM is the preferred option because it minimizes the need for optical fibers and optical wavelengths and is relatively simple and low cost to implement. It also provides an extra design dimension; a frequency plan that allows the use of low cost components but must ensure relaxed channel filtering requirements.

Low cost semiconductor lasers and photodiodes are preferred in a simple and straightforward intensity modulation direct detection system. External modulation is not appropriate because it has no significant performance benefit and has higher cost [14]. Frequency translation is relatively straightforward and cheap to implement using low cost oscillators and mixers. A low frequency reference tone can be transmitted from the central unit to the remote units to lock the oscillators to ensure accurate frequency translation.

Fig. 2 is a simplified layout diagram showing the case of four radio channels per link direction. In this example, the MIMO configuration is $2 \times 2$ ( $2 \mathrm{Tx}$ antennas and $2 \mathrm{Rx}$ antennas) and the RAU supports 2 sectors (considered appropriate for street coverage in dense urban microcell deployments), which together give the requirement for 4 radio channels per link direction. The 2 -sector DBWS base station has digital in-phase and quadrature (I/Q) inputs and outputs, consistent with open base station architectures [7, 8]. In the downlink direction, the digital $\mathrm{I} / \mathrm{Q}$ signals are converted to analog form using D/A converters and then modulated onto individual subcarriers using IQ modulators. These subcarriers are then combined and the composite signal modulates a laser diode for optical transmission to the RAU. WDM may be used to minimize the number of fibers required, especially if several RAUs must be supported from a single CU. The fiber network is shown in Fig. 1 using simple star-tree distribution, but in reality it is likely to be some form of passive optical network, based on WDM, for the support of multiple RAUs. At the RAU, the composite signal is converted back to the electrical domain using the photodiode and then split into the individual subcarriers using a splitter and appropriate filters. The IF signals are then converted into RF, for transmission over the air interface. The reverse process takes place in the 
uplink direction.

Other channels will be transmitted between CU and RAU (not shown in the figure) for control and monitoring purposes. As mentioned previously, a reference tone will also be transmitted to the RAU in order to lock the remote oscillators for accurate frequency conversion. All eight radio channels, in addition to the control channels and reference tone, can easily be accommodated in a transmission bandwidth of less than $2 \mathrm{GHz}$. An example frequency plan is shown in Fig. 3. The control channels and reference tone are not shown in this figure for the sake of clarity, but would occupy the free spectrum below 200 $\mathrm{MHz}$.

\section{B. Link Measurements}

The primary RoF link parameters are gain, equivalent input noise (EIN) and maximum input power [14]. In this section we consider the intrinsic performance of the RoF link, without electrical amplifiers (post-amplifier in the case of the downlink direction and pre-amplifier in the case of the uplink direction). Parameters for the RoF link design were obtained by constructing a link using low cost analog devices. This optical link consisted simply of a laser, an optical attenuator and a photodiode. The laser was an uncooled $1310 \mathrm{~nm}$ Emcore DFB laser [15] with a built-in isolator in a coaxial package and had a slope efficiency of $0.25 \mathrm{~W} / \mathrm{A}$ and a threshold current of $10 \mathrm{~mA}$. The maximum output power was specified at $10 \mathrm{~mW}$, which set the maximum operating current at $50 \mathrm{~mA}$. All measurements were done at this bias current. The photodiode was an Appointech $2.5 \mathrm{GHz}$ pin module [16] and had a responsivity of $1 \mathrm{~A} / \mathrm{W}$ at a wavelength of 1310 $\mathrm{nm}$. The optical attenuator was set to $10 \mathrm{~dB}$ to take the optical power budget into account. At a frequency of 1.8 $\mathrm{GHz}$ (at the upper end of the SCM frequency plan) and an optical loss of $10 \mathrm{~dB}$, the link had a gain of $-28 \mathrm{~dB}$, a P1dB of $17 \mathrm{dBm}$ and an EIN of $-131 \mathrm{dBm} / \mathrm{Hz}$. An optical power budget of $10 \mathrm{~dB}$ is expected to be adequate to take into account fiber attenuation for spans of up to $10 \mathrm{~km}$ and also to account for the splitter and connector losses in the optical network. The relatively high gain of the link was partly due to the use of a specially designed laser impedance matching transmission line [17].

The maximum input power was determined by measuring error vector magnitude (EVM) as a function of $\mathrm{RF}$ input power. This was done for various combinations of modulation complexity and number of OFDM subcarriers. EVM gives an indication of transmission quality and a minimum value is often specified in advanced radio systems with complex modulation schemes.

EVM and CNR are related through the following equation [18]:

$$
C N R=-(r+20 \log [E V M /(100 \%)])
$$

where $r$ is the peak-to-average energy ratio of the QAM constellation, and is $3.7 \mathrm{~dB}$ for $64-\mathrm{QAM}$ and $4.2 \mathrm{~dB}$ for 256-QAM. We assume a required CNR of $24 \mathrm{~dB}$ for 64QAM and $30 \mathrm{~dB}$ for 256-QAM. These values fall between the requirements for Ricean and Rayleigh propagation [13]; although the deployment scenario envisaged here is more towards Ricean (open environment), we take a slightly conservative approach. The 256-QAM CNR is extrapolated from lower modulation complexities given in [13]. For 64-QAM, the minimum EVM requirement is $4 \%$ and for 256-QAM, the minimum EVM requirement is $2 \%$.

The EVM measurements could not be performed using built-in vector signal generator (VSG) and vector signal analyzer (VSA) settings since we need user-defined combinations of QAM and OFDM subcarrier number that are not available in these units.

Real-Time Signal Transmission

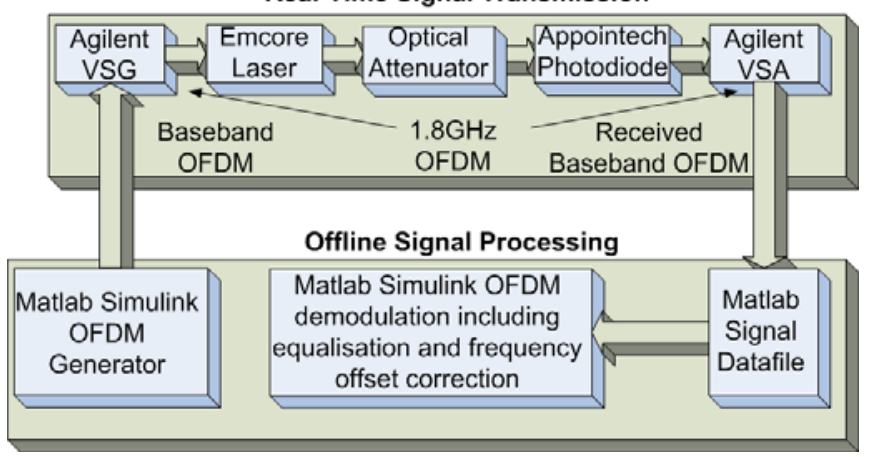

Fig. 4. User defined OFDM experimental setup.

The setup of the user defined experiments is shown in Fig. 4. For the measurements, the user defined OFDM signal was generated offline in Matlab/Simulink and downloaded to the Agilent VSG to produce a real, experimental OFDM signal at an intermediate frequency $(1.8 \mathrm{GHz})$. This user defined OFDM signal is then fed to the optical link consisting of an Emcore laser diode (1310nm), 10dB optical attenuator and an Appointech photodiode. An Agilent VSA (E4440A, with a laptop running Agilent VSA software) creates a signal data file from the OFDM signal exiting the photodiode to be processed offline in Matlab/Simulink. The offline OFDM signal processing consists of manual timing synchronization, OFDM demodulation, blind frequency offset (FO) correction and blind equalization. Frequency offset (FO) occurs when there is an unwanted frequency difference between transmitter and receiver local oscillators (LO) and causes a loss of orthogonality between the subcarriers in the OFDM signal. The blind FO technique used here finds the FO value of the received signal and applies the inverse to correct the signal.

Verification of the user defined OFDM technique was carried out through a comparison of the experimental demodulation results for a user defined OFDM signal (26.25 MSps, 128 IFFT size, 64 QAM) with those for an Agilent emulated 802.11n (26.25 MSps, 128 IFFT size, 64 
QAM), transported over an optical link ( as shown in Fig. 4). Fig. 5 shows that there is a close match between the two sets of results, verifying the accuracy of the user defined OFDM technique.

Fig. 6 shows the EVM of the RoF link as a function of the input power for 2048 IFFT size OFDM signal using 16, 64 and 256-QAM modulation at a frequency of $1.8 \mathrm{GHz}$. The IFFT (Inverse Fast Fourier Transform) block is the process by which the OFDM signals are generated and gives an upper limit to the number of subcarriers that can be used for any given block size. The results indicate insignificant difference in EVM at the same drive power for the different QAM levels. This supports the prediction of (1) that the statistical PAPR is independent of QAM level.

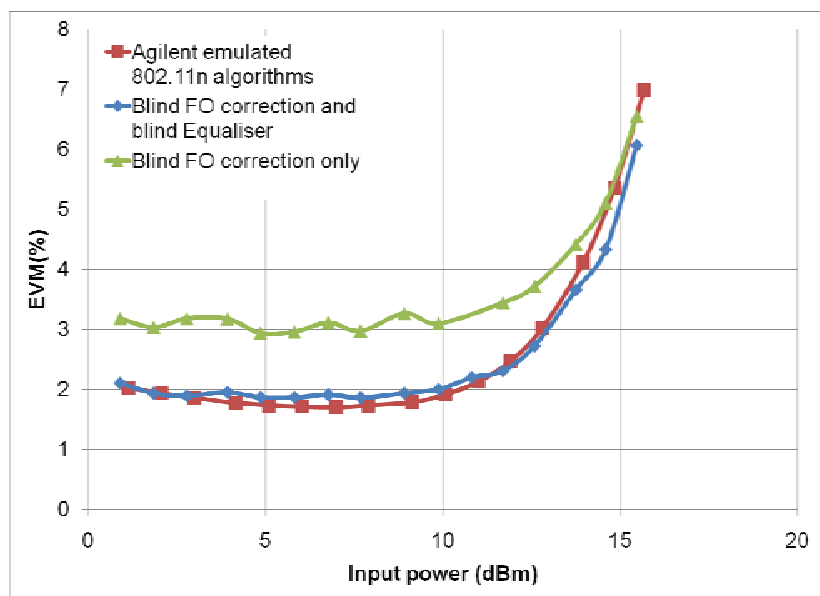

Fig. 5. Comparison of measured EVM for Agilent emulated 802.11n (26.25 MSps, 128 IFFT size, 64 QAM) signal and the user-defined emulated OFDM signal (26.25 MSps, 128 IFFT size, 64 QAM) using blind frequency offset (FO) correction and blind equalizer.

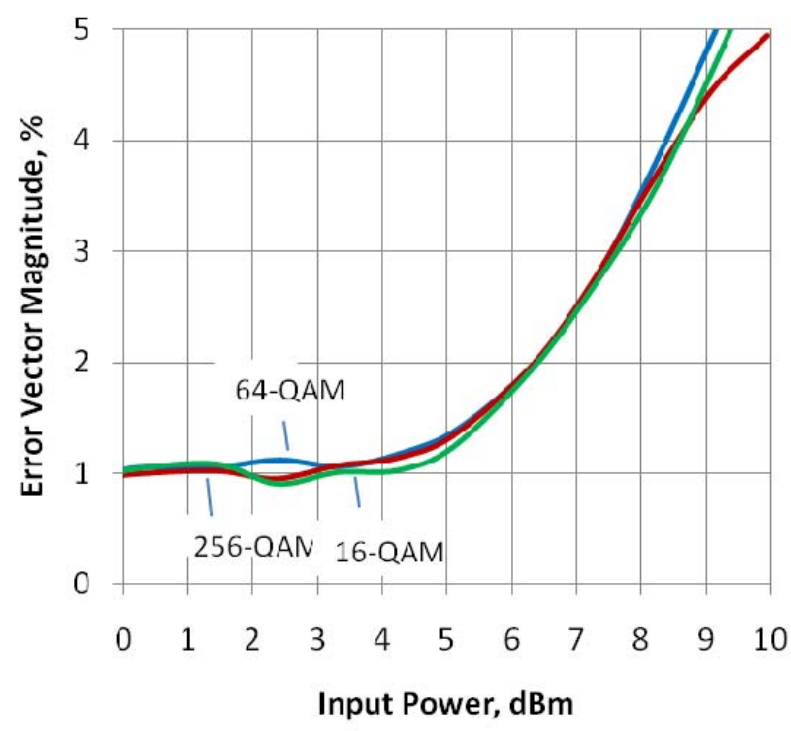

Fig. 6. EVM of the RoF link as a function of input power for 2048 IFFT OFDM signals with 16, 64 and 256-QAM modulation.

From Fig. 6 the maximum input power possible before the $2 \%$ EVM limit for 256-QAM is met is $6.4 \mathrm{dBm}$. Thus, it can be expected that for four carriers having 256-QAM modulation with 2048 IFFT size OFDM, the maximum input power per carrier will be $0.4 \mathrm{dBm}$.

Fig. 7 shows the effect of the OFDM IFFT size on the experimental RoF link. EVM is plotted as a function of input power for the case of an IFFT size of 64 and 2048. The 64 IFFT case is generated using a vector signal generator set to the IEEE 802.11g standard (64-QAM and 9Msymbols/s). The 2048 IFFT signal was generated and demodulated using the user-defined method. This signal has the same modulation level and symbol rate as the $802.11 \mathrm{~g}$ signal; the difference is due to the IFFT size. This difference is approximately $0.5 \mathrm{~dB}$ for an EVM threshold of $4 \%$ and is due to the variation of statistical PAPR as discussed earlier in section IID.

TABLE I

RADIO SYSTEM PARAMETERS

\begin{tabular}{ll}
\hline \multicolumn{1}{c}{ Parameter } & Requirement \\
\hline radio channel bandwidth & $100 \mathrm{MHz}$ \\
MIMO & $2 \times 2$ \\
sectors per RAU & 2 \\
modulation scheme & up to 256-QAM \\
number of OFDM subcarriers & up to 2048 \\
minimum approach distance & $2 \mathrm{~m}$ \\
mobile terminal transmit power (min.) & $-10 \mathrm{dBm}$ \\
mobile terminal transmit power (max.) & $+33 \mathrm{dBm}$ \\
path loss exponent & 2.0 \\
antenna gain & $10 \mathrm{dBi}$ \\
radio range target & $300 \mathrm{~m}$ \\
\hline \hline
\end{tabular}

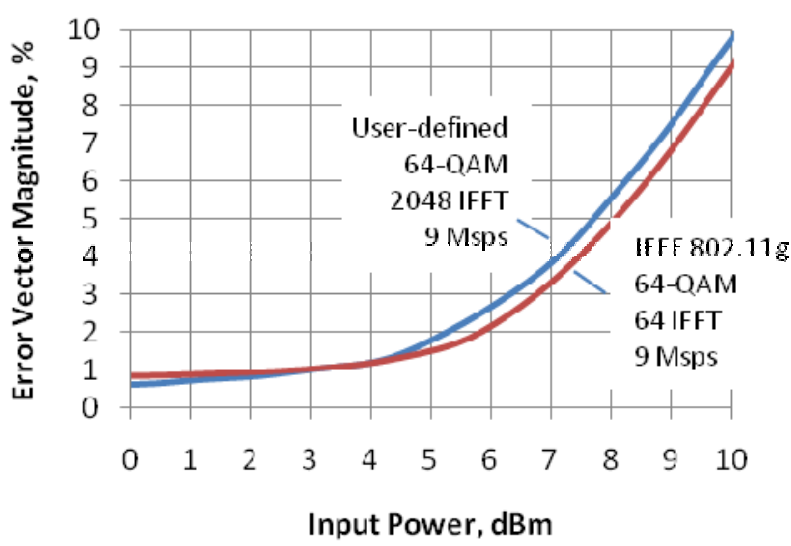

Fig. 7. EVM as a function of input power for 64 IFFT and 2048 IFFT OFDM signals with similar bandwidth and QAM level.

\section{Wireless Range Calculations}

The specifications of the remote unit amplifiers, in terms of gain, noise figure and $\mathrm{P} 1 \mathrm{~dB}$ will depend on the precise requirements and parameters of the wireless system. For the downlink direction, the amplifier gain is determined by 
the output power of the RoF link and the required transmit power at the antenna connector. This gain may need to be reduced however if the transmitter noise will otherwise be above the specified limit. For the uplink direction, a high amplifier gain gives the RoF link a relatively low noise figure. As the amplifier gain is increased, the noise figure of the link reduces until the reduction becomes less and less significant. There will be an optimum amplifier gain where the link noise figure is reduced to an acceptable level. The amplifier gain may need to be reduced however to accommodate input signals that have relatively high power. This will occur when the mobile device is close to the antenna and depends on the minimum transmit power of the mobile device and the minimum approach distance between the mobile and the antenna.

Detailed specification of next generation wireless systems is yet to be completed, however we use the FUTON DBWS here to provide an example system for RoF link design. This is accomplished by taking the intrinsic RoF link parameters given above and then calculating the remote unit amplifier gains from these parameters and from the DBWS specifications. The signal power and CNR levels can then be calculated at the link output, for both uplink and downlink directions. The maximum path loss can then be calculated; this then leads to wireless range calculations by assuming a particular propagation environment.

The purpose of this work is to analyze the performance degradation from the RoF link by comparing the wireless range with and without the link. We therefore use a simple path loss model with a single path loss exponent for the analysis. An open environment with a path loss exponent of 2 is used for calculating wireless range. We also assume the use of directional antennas with gains of $10 \mathrm{dBi}$. These assumptions are based on a deployment scenario where we have coverage along streets in city centers from antennas mounted on items of street furniture such as lamp posts and on the sides of buildings (a typical DAS microcell deployment scenario).

DBWS specifications and other assumptions used in the wireless range calculations are given in Table 1. For most wireless systems, including DBWS, the uplink direction is the limiting factor in determining wireless range. This is due to the relatively low transmit power from the mobile terminal, as a result of the need to maximize battery life. We therefore concentrate on this direction in the following analysis.

Maximum path loss in the uplink direction is the difference (in $\mathrm{dB}$ ) between the transmit power of the mobile terminal and the receiver sensitivity of the base station, taking antenna gain into account. When a RoF link is placed between the receive antenna and the base station receiver, the effect is to decrease the sensitivity as a result of the high noise figure of the RoF link. The noise figure of the RoF link can be calculated from the link gain and output noise power, using the relation:

$$
F=N_{\text {out }} /(k \cdot T \cdot B . G)
$$

where $F$ is the noise factor, $N_{\text {out }}$ is the total output noise power, $k$ is Boltzmann's constant, $B$ is the noise bandwidth and $G$ is the link gain. The noise figure is simply the noise factor expressed in $\mathrm{dB}$. Using this relation, the noise figure of the RoF link becomes $43 \mathrm{~dB}$. An electrical preamplifier is used to reduce this noise figure to an acceptable level and the overall noise figure is given by the Friis' formula [19] for cascaded noise sources. The gain of the preamplifier must be high enough to reduce the overall noise figure, but is limited by the need to handle high input powers from the receive antenna. The maximum input power at the amplifier input is determined by the minimum approach distance between antenna and mobile terminal and by the minimum transmit power of the mobile terminal. For the parameters given in Table 1, this maximum input power is $49.3 \mathrm{dBm}$ at the antenna connector. The maximum amplifier gain becomes $49.7 \mathrm{~dB}$ since the maximum input power to the RoF link is $0.4 \mathrm{dBm}$ per carrier for the worst case of 256-QAM modulation. Assuming an amplifier noise figure of $5 \mathrm{~dB}$ and a base station receiver noise figure of $5 \mathrm{~dB}$, the overall noise figure becomes $5.3 \mathrm{~dB}$. The RoF link therefore degrades the noise figure by only $0.3 \mathrm{~dB}$.

The receiver sensitivity in $\mathrm{dBm}, S_{r x}$, is calculated from the following relation:

$$
S_{r x}=-174+B+C N R+N F_{t}+L_{i m p l}
$$

where -174 is the thermal noise power $(k \cdot T$, in $\mathrm{dBm} / \mathrm{Hz})$, $B$ is $10 \log$ (noise bandwidth), CNR is the carrier to noise ratio required for successful demodulation of the signal, $N F_{t}$ is the total noise figure and $L_{\text {impl }}$ is the implementation loss of the receiver. For the baseline case (no RoF link), the receiver sensitivity is $-60 \mathrm{dBm}$ for 64-QAM using a receiver noise figure of $5 \mathrm{~dB}$ and a receiver implementation loss of $5 \mathrm{~dB}$. For $256-\mathrm{QAM}$, the sensitivity is $-54 \mathrm{dBm}$. The maximum path loss is therefore $103 \mathrm{~dB}$ (64-QAM) and 97 $\mathrm{dB}$ (256-QAM) for the parameters given in Table 1. This path loss can support a wireless range of $960 \mathrm{~m}$ (64-QAM) and $480 \mathrm{~m}$ (256-QAM) for a path loss exponent of 2 (open, line-of-sight environment). The RoF link desensitizes the receiver by $0.3 \mathrm{~dB}$ and the wireless range becomes $930 \mathrm{~m}$ (64-QAM) and $470 \mathrm{~m}$ (256-QAM). It must be noted that this analysis does not take into account the effects of fiber dispersion and fiber non-linearities and therefore the noise figure desensitization is likely to be higher for long fiber spans. However, typical fiber spans for the deployments being considered for these systems are of the order of a few kilometers so we do not expect a significant impact on our analysis. 


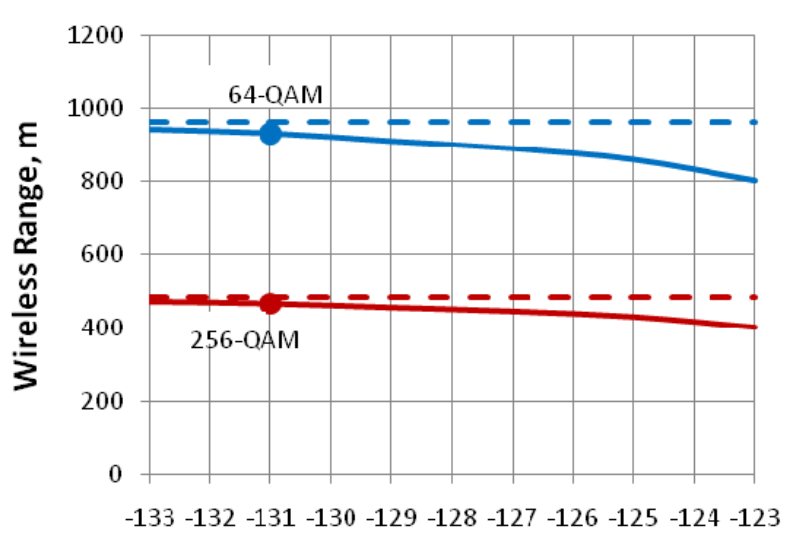

Equivalent Input Noise, $\mathrm{dBm} / \mathrm{Hz}$

Fig. 8. Wireless range as a function of RoF link EIN for 64-QAM and 256-QAM modulation. The baseline range values (without RoF link) are shown as dashed lines.

The effect of RoF link noise is shown in Fig. 8, where wireless range is plotted as a function of link EIN. This is done for both 64-QAM and 256-QAM modulation and the baseline wireless ranges (with no RoF link) are added as dashed lines. The performance of the experimental RoF link for each modulation type is shown in this figure as points. It is clear that the RoF link has minimal impact on wireless range for the example wireless system used here for EIN values below $-125 \mathrm{dBm} / \mathrm{Hz}$. The good performance of the RoF link is largely due to the wide range of uplink power control in the wireless system and the assumption that there will be no uncoordinated mobile terminals using the same frequency band. This assumption is considered to be valid because the channel bandwidth is very wide and therefore competitor systems are unlikely to be using the same frequency band. This power control allows the electrical preamplifier in the RAU to have very high gain, which in turn means that the overall noise figure can approach the baseline value.

\section{AnAlog or Digital Transmission?}

The preceding analysis has shown that RoF links give a relatively low level of degradation to the radio range of an example next generation wireless system, even though the links must support wide channel bandwidths, high modulation complexity and multiple MIMO channels. The falling costs of high-speed D/A and A/D converters, has led to heightened recent interest in digitized radio over fiber links [20, 21]. In fact, as stated in Section I, such digital links have been specified by CPRI and OBSAI for current wireless systems (UMTS, WiMAX and LTE) to connect digital base stations to remote radio heads. These links transmit digital I and Q samples of the radio signal which means that the remote radio heads can be very simple, consisting of $\mathrm{D} / \mathrm{A}$ converters, upconverters and amplifiers for the downlink direction and $\mathrm{A} / \mathrm{D}$ converters, downconverters and amplifiers for the uplink direction. All modulation functions take place in the central unit. It is very important that the remote radio heads are small and relatively simple so that they can be located in places where space is limited. This is a similar architecture to that described in this paper, so it is important to consider which link type is better for this application.

The link design described above has been produced to minimize the number of optical fibers needed to connect a central unit with multiple remote units. In order to compare like with like, a digital link design must meet the same requirements. We therefore consider a time division multiplexed (TDM) serial digital link as an alternative to the SCM RoF link for this application [22]. In terms of performance it is clear that there is little difference between the two options since the radio range with the RoF link is only marginally less than the case without the link. The main consideration therefore becomes cost, i.e., which is the most cost effective option. Fig. 9 shows the simplified layouts of each approach. All functions are common to both approaches (though they may have different locations) apart from the optical transceivers and the multiplexers/demultiplexers, shown as the shaded boxes in Fig. 9. We therefore consider the cost difference between these items in this analysis.

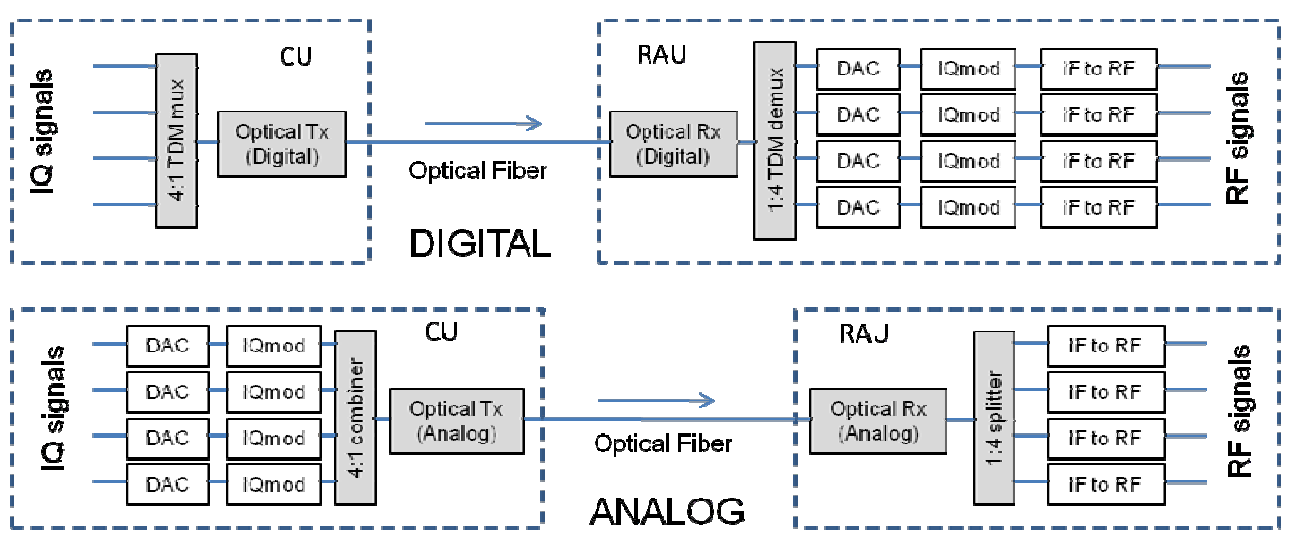

Fig. 9 Block diagram showing simplified layouts of analog and digital transmission link options (downlink direction only). The 
shaded boxes denote the components which differ between the two options.

CPRI and OBSAI specify digital links with bit rates up to $6144 \mathrm{Mb} / \mathrm{s}[7,8]$, which is considered to be adequate for transporting a small number of $20 \mathrm{MHz}$ radio channels. Using OBSAI as an example, we extrapolate a sampling rate requirement of $153.6 \mathrm{MHz}$ for a $100 \mathrm{MHz}$ radio channel from the requirement in the specification for a $20 \mathrm{MHz}$ radio channel [23]. For I and Q sample widths of 16 bits, the bit rate requirement becomes $4915 \mathrm{Mb} / \mathrm{s}$ for LTE. Note that this is a conservative estimate since the sample widths can be up to 20 bits for LTE [7]. Taking other requirements into account such as control and management channels, it is expected that the maximum CPRI/OBSAI bit rate of $6144 \mathrm{Mb} / \mathrm{s}$ will only support a single $100 \mathrm{MHz}$ radio channel. Four $100 \mathrm{MHz}$ radio channels will therefore require a serial bit rate of more than $24 \mathrm{~Gb} / \mathrm{s}$. This must be mapped on to an existing low cost optical transmission standard such as Ethernet, and in this instance will require $40 \mathrm{~Gb} / \mathrm{s}$ Ethernet $(40 \mathrm{GbE})$. $40 \mathrm{GbE}$ optical transceivers are not available at present but their cost can be estimated from the cost of current $10 \mathrm{GbE}$ optical transceivers by applying a predicted cost ratio. This ratio is predicted to be 3.4 by 2012 [24]. 10GbE-LR optical transceivers (LR denotes a fiber span capability of up to $10 \mathrm{~km}$ ) cost approximately $\$ 2000$ in low volume at present [22]. A bidirectional optical link will require two transceivers so the total cost for a bidirectional $40 \mathrm{GbE}$ link becomes $\$ 13600$ after applying the predicted cost ratio. For the case of the RoF link, a pair of $2 \mathrm{GHz}$ optical transceivers with adequate performance will cost approximately $\$ 2000$ [22].

The cost difference between optical transceivers for RoF and digital transmission is obviously very significant when a number of wideband radio channels must be transported. However, even more significant is the cost difference between the multiplexing equipment for each case. The digital link will require $40 \mathrm{~Gb} / \mathrm{s} 4: 1$ and 1:4 multiplexers and demultiplexers. These cost approximately $\$ 7000$ each in low volume [22], so the total cost of mux/demux is $\$ 28000$ for the digital case. For the case of the RoF link, simple low frequency (less than $2 \mathrm{GHz}$ ) power splitters, combiners and filters are all that are required. These are estimated to cost no more than approximately $\$ 800$ in total. The combined cost for digital transmission and multiplexing is $\$ 41600$ compared to the cost for RoF transmission and multiplexing of $\$ 2800$. Note that these figures are for low volumes and that in practice the cost difference will be lower than this. However it is expected that the cost difference will still be highly significant between the two options.

It is clear that RoF is the better option for the transport of multiple wideband radio channels because its performance has marginal impact on the radio range while it offers significantly lower implementation cost. However it must be noted that this result is specific to the case where we need to transport multiple wideband radio channels and is not generally applicable to a comparison between analog and digital transmission systems.

\section{CONCLUSION}

A radio over fiber link design has been described for distributed antenna applications in next generation wireless systems having wide radio channel bandwidth, complex modulation, a high number of OFDM subcarriers and several MIMO channels. This design uses subcarrier multiplexing to carry these channels in order to minimize cost and to conserve wavelengths.

The performance of the radio over fiber links is shown to be acceptable using low cost lasers and photodiodes. We calculate a base station receiver desensitization of only 0.3 $\mathrm{dB}$ for an experimental link constructed using readily available components, which ensures that the impact on uplink wireless range is minimal. This is a consequence of the uplink power control in the wireless system, which allows the uplink amplifier gain to be high enough to reduce the overall noise figure significantly.

We have also compared the use of radio over fiber to an approach that uses digitized transmission and found that the digital links are at least an order of magnitude more expensive, as a result of the high line rates that the digital links require for wideband radio channels.

\section{ACKNOWLEDGMENT}

The authors would like to thank I. Garcia Zuazola (Univ. Kent) for designing and constructing the laser matching circuit used in this work, S. Pato and J. Pedro (both NSN, Portugal) for the line rate requirement analysis for wideband digitized RF links and also P. Assimakopolous (Univ. Kent) for his help with the peak to average power ratio analysis for OFDM signals.

\section{REFERENCES}

[1] A.J. Cooper, "Fibre/radio for the provision of cordless/mobile telephony services in the access network", Electronics Letters, vol. 26, Nov. 1990

[2] D. Wake, M. Webster, G. Wimpenny, K. Beacham and L. Crawford, "Radio over fiber for mobile communications", Int. Topical Meeting on Microwave Photonics, MWP'04. Oct. 2004.

[3] M. Fabbri and P. Faccin, "Radio over Fiber Technologies and Systems: New Opportunities", Int. Conf. Transparent Optical Networks, ICTON '07. vol. 3, July 2007

[4] M. Döttling (Ed.), W. Mohr (Ed.), A. Osseiran (Ed.) "Radio Technologies and Concepts for IMT-Advanced”, Wiley, 2009.

[5] www.3gpp.org

[6] S. Pato, P. Monteiro, N. Gomes, A. Gameiro, T. Kawanishi, "NextGeneration Distributed and Heterogeneous Radio Architectures: the FUTON Project", Asia-Pacific Microwave Photonics Conference, Beijing, China, April, 2009.

[7] "Common Public Radio Interface; Interface Specification", v4.1, 2009, available at www.cpri.info

[8] "Open Base Station Architecture Initiative; BTS System Reference Document", v2.0, 2006, available at www.obsai.com 
[9] M. Weiss, M. Huchard, A. Stohr, B. Charbonnier, S. Fedderwitz and D. Jager, "60-GHz Photonic Millimeter-Wave Link for Short- to Medium-Range Wireless Transmission Up to $12.5 \mathrm{~Gb} / \mathrm{s}$ ", J. Lightwave Tech., vol. 26, Aug. 2008.

[10] H. Ochiai, H. Imai, "On the distribution of the peak-to-average power ratio in OFDM signals," IEEE Trans. Commun, Vol. 49, Feb. 2001.

[11] R. Baxley, G. T. Zhou, "Power saving analysis of peak-to-average power ratio reduction in OFDM," IEEE Trans. Consum. Elec., vol. 50, August 2004.

[12] P.Assimakopoulos, L. Vieira, A. Nkansah, D. Wake, N.J. Gomes, and F. van Dijk, "Modelling of a DFB laser at low bias directly modulated with an OFDM signal for RoF applications", IEEE Int. Topical Mtg. on Microwave Photonics, MWP 2009, Valencia, Spain, Oct. 2009

[13] EN 300744 v1.1.2, "Digital Video Broadcasting; Framing structure, channel coding and modulation for digital terrestrial television", 2008.

[14] D. Wake, A. Nkansah, N. J. Gomes, G. de Valicourt, R. Brenot, M. Violas, Z. Liu, F. Ferreira and S. Pato, "A Comparison of Radio over Fiber Link Types for the Support of Wideband Radio Channels", submitted to J. Lightwave Tech. special issue on very high throughput wireless sytems, 2010.

[15] Emcore model 1935F datasheet, available at www.emcore.com

[16] Appointech 2.5Gbps InGaAs PIN photodiode module datasheet, available at www.appointech.com

[17] I. Garcia Zuazola, J. Batchelor, A. Nkansah, D. Wake and N. Gomes, "Microcell MIMO RoF system using low cost array antennas and improved DFB matching lines", Loughborough Antennas \& Propagation Conference (LAPC), November 2009.

[18] W. Music, TG3 measurement accuracy, IEEE P802.15 working group for wireless personal area networks, 2001.

[19] H. Friis, "Noise figure of radio receivers", Proc. of the IRE, vol. 32, July 1944.

[20] A. Nirmalathas, P.P. Gamage, Y. Yang, and C. Lim, "Cost-effective optical backhaul for broadband wireless", Invited Paper, Proc. IEEE Photonics Society Annual Meeting, Belek-Antalya, Turkey, October, 2009.

[21] T.Li, M. Crisp, R. Penty, and I. White, "Low bit rate digital radio over fibre system", IEEE Int. Topical Mtg. on Microwave Photonics, MWP 2009, Valencia, Spain, Oct. 2009

[22] D. Wake, S. Pato, J. Pedro, E. López, N. Gomes and P. Monteiro, "A Comparison of Remote Radio Head Optical Transmission Technologies for Next Generation Wireless Systems", Proc. IEEE Photonics Society Annual Meeting, Belek-Antalya, Turkey, October, 2009.

[23] FUTON project Deliverable D5.2, "Initial predicted performance of radio over fiber distribution network", 2008, available at www.ictfuton.eu

[24] K. Hiramoto, M. Shishikura and M. Traverso, "Demonstration of $40 \mathrm{~Gb} / \mathrm{s} 1310 \mathrm{~nm}$ EA- DFB Laser feasibility for $40 \mathrm{GbE} 10 \mathrm{~km}$ SMF application" IEEE 802.3ba Task Force presentation, Korea, Sept. 2008 . 
David Wake received the B.Sc. degree in applied physics from the University of Wales, Cardiff, U.K., in 1979, and the Ph.D. degree from the University of Surrey, Surrey, U.K., in 1987. He is currently a Senior Research Fellow with the University of Kent, Canterbury, U.K.

From 2003 to 2005, he was Director of Research and Development and Chief Scientist with Microwave Photonics Inc., a startup company based in Los Angeles, CA, which was formed to develop a product set for the mobile communications industry based on novel radio-over-fiber technology. In 2002, he cofounded Zinwave Ltd., a startup company aimed at exploiting innovative radio-over-fiber technology for the mobile communications industry. He has been involved in the radio-over-fiber research topic for approximately 18 years, initially with BT Laboratories, where he was the Program Manager for the microwave photonics research domain and then with University College London as a Senior Research Fellow.

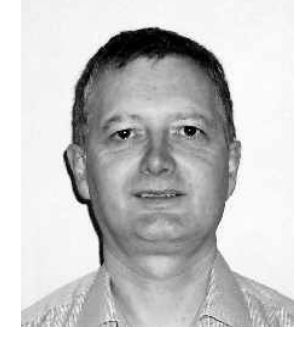

Anthony Nkansah received the B.Eng. (with honors) degree in electronic engineering, the M.Sc. degree in broadband and mobile communication networks and Ph.D degree in electronic engineering from the University of Kent, Canterbury, U.K., in 2000, 2001 and 2007, respectively. His research interests include low-cost microwave and millimeter-wave radio-over-fiber networks and their deployment within premises.

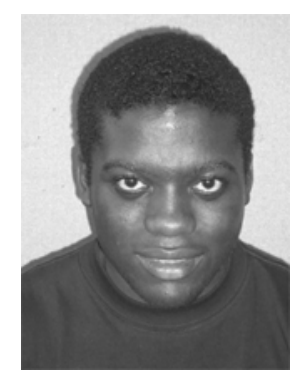

Nathan J. Gomes (M'92 - SM'06) received the BSc degree from the University of Sussex, UK, in 1984 and the PhD degree from University College London in 1988, both in electronic engineering.

From 1988 to 1989 he held a Royal Society European Exchange Fellowship at ENST, Paris. Since late 1989, he has been at the University of Kent, where he is now a Reader in Broadband Communications. His present research interests include radio over fiber systems and networks, the photonic generation and transport of millimeter-wave signals, and photoreceivers for such applications.

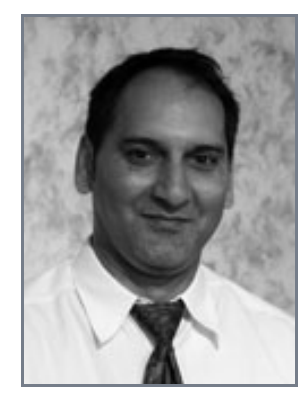


> JLT-12081-2009.R1< 